\title{
The Influence of God Class and Long Method in the Occurrence of Bugs in Two Open Source Software Projects: An Exploratory Study
}

\author{
Aloisio Sampaio Cairo * Glauco de Figueiredo Carneiro * \\ Antônio Maria Pereira de Resende ${ }^{\star} \quad$ Fernando Brito e Abreu \\ ^ Universidade Salvador (UNIFACS), Brazil Universidade Federal de Lavras (UFLA), Brazil \\ ${ }^{\text {in }}$ Instituto Universitário de Lisboa (ISCTE-IUL), Portugal
}

\begin{abstract}
Context: Code smells are associated with poor design and programming style that often degrades code quality and hampers code comprehensibility and maintainability.

Goal: In this paper, we investigated to which extent classes affected by the God Class and Long Method code smells were more susceptible to the occurrence of software bugs.

Method: We conducted an exploratory study targeting two well known open source software projects, Apache Tomcat and Eclipse JDT Core Component. We applied correlation analysis in order to evaluate to which extent Long Method and God Class were related to the occurrence of bugs.

Results: We have found a significant correlation of Long Method and Commits and, on the other hand, a poor correlation of God Class and Commits in the two analyzed projects. Therefore, we expected that the higher the number of occurrences of Long Method, the higher the chances of more commits in a class that contains this method, which could result in the increase of occurrence of bugs.

Conclusion: Based on the results, we confirmed what other studies pointed out, regarding classes affected by Long Method being more bug-prone than others. In practice, we found evidence, from analyzed data, that the occurrence of Long Method implies more effort in maintenance tasks.
\end{abstract}

\section{Introduction}

Throughout releases, a software project usually includes new functionalities and fixes bugs that were found and considered relevant in previous releases. Since maintenance costs are the highest share in the development lifecycle [1], special attention should be dedicated to avoid bugs.

Martin Fowler, in his book on software refactoring [2], claimed that code smells can negatively impact software quality, namely on its understandability and reliability. For more than a decade after that, several authors have claimed that the empirical evidence of such impact was still scarce [3, 4, 5] and, as such, Fowler's claim could not be considered as fully confirmed. Among the 22 code smells presented in Fowler's book, we selected God Class and Long Method to provide further evidence of their influence on software bugs occurrence. The former refers to classes that centralize the intelligence of the system, realizing too much work, when compared with the remainder classes, while the latter refers to methods with many statements and/or complex control flow structures [6].

To reach our stated research objective, we analyzed two projects, Apache Tomcat and Eclipse JDT Core Component. Tomcat is a "pure Java" HTTP web server environment in which Java code can run. JDT (Java Development Tools) Core Component is the Java infrastructure of Eclipse's Java IDE. We have chosen these two projects because they: (i) are open source; (ii) have a prominent popularity in the Java community; (iii) have been studied in the literature by several authors [7, 8, 9]; (iv) are related in the scope of this study.

Regarding the last reason, we found the relationship in their bug repository: 51 closed bugs from Apache Tomcat are related to the Eclipse project. These bugs were reported in classes affected by God Class or containing methods affected by Long Method in the following releases analyzed in this study: 9_0_9 of (Apache Tomcat) and Y20180725-2200 (Eclipse JDT Core). Moreover, we have also noticed that 36 bugs out of those 51 were reported in classes simultaneously affected by both code smells in those releases. Hence, we considered that the influence of the aforementioned code smells in the selected software projects deserved further analysis.

The remainder of this paper is laid out as follows. Section 2 overviews previous related research work. The experimental design of our study is presented in Section 3 and Section 4 discusses its results. Finally, in Section 5 , we present concluding remarks and future work. 


\section{Related Work}

Lehman's second law of software evolution [10] argues that as a project grows, software complexity increases as well. Constantly changing code is the context in which code smells find favorable conditions to arise. Studies have reported that code smells usually affect a software entity, such as class or method, after a change executed to fix a bug [11]. To tackle this problem, several approaches have been proposed to support the identification of code smells, as described in secondary studies such as the ones conducted by Rasool and Arshad [12] and Fernandes et al. [13].

According to [14], code smells are indicators of poor source code quality and hamper its maintenance and reuse. The influence of the God Class and Long Method code smells on software bugs occurrence has been studied by several authors. Li and colleagues [15] reported the influence of Shotgun Surgery, God Class, and God Method code smells on bugs based on the analysis of post-release system evolution. They confirmed that some smells were positively associated with the class error probability in three errorseverity levels (High, Medium, and Low), usually applied to classify issues. In [16], the authors unveiled the relationship among Shotgun Surgery, God Class and God Methods code smells and the occurrence of bugs in three Eclipse releases (3.0, 2.1 and 2.0). Olbrich and colleagues [17] presented evidence that instances of God Class and Brain Class suffered more frequent changes and contained more defects than classes not affected by those smells.

Later, Nascimento and SantAnna [18] analyzed five software projects (Apache Ant, Apache Jmeter, Apache Lenya, Apache Tomcat and Apache Xerces) to conclude that classes affected by the code smells Data Class, Data Clumps, Feature Envy, God Class, Message Chain, Schizophrenic Class and Tradition Breaker had a higher likelihood to introduce bugs than other classes from the same project. They further reported God Class as the smell with the greatest number of related bugs in the analyzed projects with a percentage of $20 \%$, followed by Feature Envy with a percentage close to $15 \%$, Schizophrenic Class with $9 \%$ and Message Chains with 7\%. In addition, Palomba and colleagues [8] claimed that classes with code smells tend to be more change- and fault-prone than other classes and that this is even more noticeable when the same class is affected by multiple smells.

A recent systematic literature review conducted by Cairo and colleagues [19] claims that there is evidence in the literature showing that software entities, such as classes affected by code smells, tend to be more prone to change and failure than other classes.

\section{The Experimental Design}

This section describes the characteristics and steps applied in this exploratory study. We adopted the Goal-
Question-Metric (GQM) approach [20], as described in Table 1. to derive the following Research Question (RQ): To which extent God Class and Long Method code smells influence the occurrence of bugs in Apache Tomcat and Eclipse's JDT Core Component projects?

Table 1: Research goal based on the GQM approach

\begin{tabular}{|l|l|}
\hline Analyze & $\begin{array}{l}\text { the God Class and Long Method } \\
\text { code smells }\end{array}$ \\
\hline for the purpose of & evaluating their influence \\
\hline with respect to & the occurrence of bugs \\
\hline from the viewpoint of & maintainers and developers \\
\hline in the context of & $\begin{array}{l}\text { Apache Tomcat and Eclipse's } \\
\text { JDT Core Component } \\
\text { open source software projects }\end{array}$ \\
\hline
\end{tabular}

To reduce the collection time and the frequent errors that arise during code smells manual detection [21], we used the PMD static code analyzer. We performed the six steps represented in Figure 1 that will be further detailed in the following paragraphs, using a common template (data input, step description, performing the step, data output).

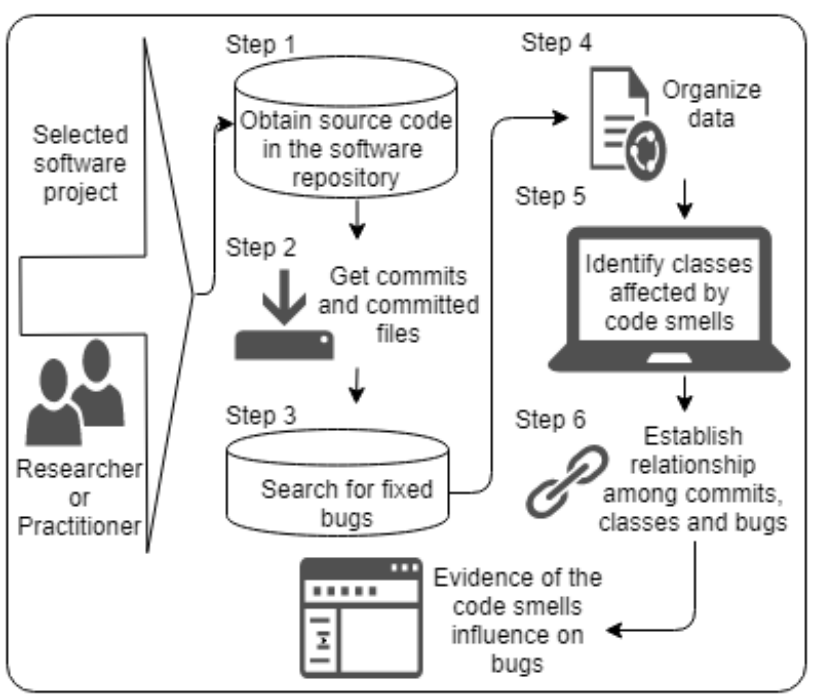

Figure 1: Proposed approach to analyze the influence of code smells on bugs occurrence in a software project

\subsection{Step 1 - Obtain source code}

Data input: URL of selected software projects repository. Step description: We cloned the sources of each selected software project using Git Bash, a Windows application which provides a Git command line shell. Performing the step: We executed the git clone command. Data output: Local repository containing the source code of selected software projects. 


\subsection{Step 2 - Get commits and coresponding files}

Data input: GitHub features that allow the collection of data related to commits performed in a software project.

Step description: We executed the command lines 1 and 2 presented below using Git Bash. Besides commits, we also collected the files involved in each commit.

Performing the step: Execute the commands as follows:

(1) git log -pretty=format:"\%H \%h \%an \%ad \%s" grep $="$ bug" $>$ log-commit.txt (stores the names of all files involved in each commit in the named file)

(2) git show hash-numbers - pretty=format: "\% $\mathrm{H}^{\prime \prime}-$ stat $>$ log-arquivos.txt (stores all commit hashes in the named file)

\subsection{Step 3 - Search for fixed bugs}

Data input: Bugzilla features that allow the collection of data related to bugs of a specific software project.

Step description: Identify all bugs marked as closed for all analyzed software projects.

Performing the step: We collected all closed bugs for Apache Tomcat until August 8th (2018) and for Eclipse until September 9th (2018). We used the Bugzilla's REST API to obtain data from the projects' repositories.

Data output: Files containing information related to bugs in JSON format from the analyzed software projects.

\subsection{Step 4 - Organize data}

Data input: Data about commits and bugs for each project. Step description: We tabulated raw data to prepare them for statistical analysis.

Performing the step: All data was inserted in a database and we applied queries to get it organized in table format. After that, we exported them to a spreadsheet for applying statistical analysis. An automatic routine was implemented to capture the ID from Bugzilla repositories. Only Java files were considered and all others (e.g. configuration files) were deleted, since only the former were relevant for code smells detection. We also did not consider commits in files not related to code smells. Some long absolute paths to Java classes were incomplete, such as, for instance in ".../apache/catalina/connector/CoyoteAdapter.java". In those cases, we searched manually for the file and completed the correct absolute path string, being careful in cases of multiple files with the same name, located in different packages.

Data output: Commit data, file involved in each commit, and bugs of each project.

\subsection{Step 5 - Identify classes affected by code smells}

Data input: Source code of projects cloned in step 1.

Step description: We used the PMD tool to collect information about code smells.

Performing the step: The PMD tool was applied in each project to detect God Class and Long Method occurrences. Data output: A table containing all software project classes affected by God Class and Long Method, and the number of occurrences of each code smell, for each class.

\subsection{Step 6 - Evidence of code smells influence on bugs}

Data input: Each line of tabulated data contains software project name, version, absolute path of Java class, class name, occurrences of Long Method, occurrences of God Class and total amount of commits involving both projects. Step description: We performed statistical analysis on available data to check the intensity of correlations among code smells and bugs.

Performing the step: Since the data was not normally distributed, we used the Spearman non-parametric correlation coefficient to check the correlation among the occurrences of each code smell and the amount of commits. We grouped the commits in ranges to check the correlation. Data output: Intensity of correlation among code smells occurrences and amount of bugs, represented by commits.

\section{The Study Results}

\subsection{Distribution adherence testing}

All data used in this analysis is available in a public Github repository 1 The total number of classes analyzed with at least one commit are 1049 and all those classes have at least one code smell. The first step of a statistical correlation analysis is to verify whether the data is normally distributed. This verification allows the selection of the most suitable statistical technique to be applied. We used the EasyFit too ${ }^{2}$ to perform the aforementioned verification. The results pointed out that the occurrences of Long Method and God Class have Poisson Distribution and the amount of commits has Geometric distribution. Since none of them has a normal distribution, that required the application of a non-parametric correlation coefficient such as Spearman's.

\subsection{Correlation analysis by commit ranges}

The main research question stated in Section 3 aimed at devising to which extent God Class and Long Method code smells influence (or have impact in) the occurrence of bugs in two open source projects. In this study we only considered bugs that were fixed and took as surrogate of those bugs the number of file (class) commits they cause. Therefore, the research question originated two sub-questions, one per each code smell, where we want to check if there is a statistical significant association among the variables that allow us to not discard that the aforementioned impact exists. In other words, the sub-questions are:

a) Is there a correlation between the occurrences of Long Method in a class and the amount of commits in that class?

\footnotetext{
${ }^{1}$ https://github.com/SEKE2019CodeSmell/SEKE2019EvidenceCodeSmells

${ }^{2} \mathrm{http}: / / \mathrm{www} \cdot \mathrm{mathwave.com/}$
} 
b) Is there a correlation between the occurrences of God Class in a class and the amount of commits in that class?

To answer those questions, while eliminating some random deviations that might have occurred in collected data, we grouped the analyzed classes by consecutive ranges of 3 commits. Therefore, the first range contains classes with commits within the $[0,3[$ range, the second in the $[3,6[$ range, and so forth, as represented in the first two columns in Tables 2 and 3 for the Apache Tomcat and Eclipse JDT Core Component, respectively. For instance, the values of 130 and 279 on the first line of column II of the same tables represent the total number of classes with 0,1 or 2 commits (range defined in column I) in the two analyzed software projects. We have also normalized the amount of class commits, occurrences of Long Method and God Class, by calculating their average value per class. The column labels of Tables 2 and 3 are as follows:

(I) Commit range ( $>=$ and $<$, respectively)

(II) Class total

(III) Commits Sum

(IV) Long Method Total (occurrences)

(V) God Class Total (occurrences)

(VI) Code Smells Total (occurrences)

(VII) Average Commits Per Class

(VIII) Long Method Average (occurrences) Per Class

(IX) God Class Average (occurrences) Per Class

The highlighted lines in Tables 2 and 3 represent the data that we considered as relevant. The classes with commits above the 57-60 range were clear outliers and were ignored in the following study. To confirm this claim, we generated dispersion charts (Figures 2 and 3) to visually check the distribution of data in the ranges. The Long Method chart provides some evidence of correlation, because the points are around the tendency line and increasing, meaning a positive correlation. Furthermore, it is possible to obtain a curve with even better fit (as measured by the R squared coefficient of determination), what suggests that the effect of this code smell on commits may be non-linear. As for the God Class the chart does not provide evidence of correlation. After determining the valid data, without irrelevant lines and outliers, we calculated the Spearman correlations. The results are presented in Table 4.

To interpret the values of the coefficients in Table 4. we used the ordinal intensity scale presented in Table 5 (from [22]). Therefore, based on the obtained values of the Spearman correlation coefficient in Table 4 we can state that there is a very high positive correlation between Long Method Average and Commit Average. Consequently, that result allow us to raise the hypothesis that the number of commits in a class may be impacted by the occurrences of the Long Method code smell. The same cannot be claimed for the God Class code smell, since only a moderate positive correlation was observed. For the Eclipse project, we
Table 2: Classes grouped by commit ranges (APACHE TOMCAT)

\begin{tabular}{|c|c|c|c|c|c|c|c|c|c|}
\hline (I) & & (II) & (III) & (IV) & (V) & (VI) & (VII) & (VIII) & (IX) \\
\hline 0 & 3 & 130 & 105 & 47 & 109 & 156 & 0,81 & 0,36 & 0,84 \\
\hline 3 & 6 & 71 & 282 & 27 & 64 & 91 & 3,97 & 0,38 & 0,90 \\
\hline 6 & 9 & 47 & 330 & 16 & 42 & 58 & 7,02 & 0,34 & 0,89 \\
\hline 9 & 12 & 23 & 237 & 7 & 24 & 31 & 10,30 & 0,30 & 1,04 \\
\hline 12 & 15 & 22 & 283 & 14 & 22 & 36 & 12,86 & 0,64 & 1,00 \\
\hline 15 & 18 & 14 & 226 & 11 & 14 & 25 & 16,14 & 0,79 & 1,00 \\
\hline 18 & 21 & 12 & 229 & 6 & 11 & 17 & 19,08 & 0,50 & 0,92 \\
\hline 21 & 24 & 8 & 177 & 9 & 9 & 18 & 22,13 & 1,13 & 1,13 \\
\hline 24 & 27 & 7 & 171 & 9 & 7 & 16 & 24,43 & 1,29 & 1,00 \\
\hline 27 & 30 & 1 & 27 & 1 & 1 & 2 & 27,00 & 1,00 & 1,00 \\
\hline 30 & 33 & 4 & 122 & 3 & 4 & 7 & 30,50 & 0,75 & 1,00 \\
\hline 33 & 36 & 1 & 35 & 4 & 1 & 5 & 35,00 & 4,00 & 1,00 \\
\hline 36 & 39 & 2 & 74 & 4 & 2 & 6 & 37,00 & 2,00 & 1,00 \\
\hline 39 & 42 & 1 & 39 & 3 & 1 & 4 & 39,00 & 3,00 & 1,00 \\
\hline 42 & 45 & 1 & 42 & 4 & 1 & 5 & 42,00 & 4,00 & 1,00 \\
\hline 45 & 48 & 1 & 46 & 3 & 1 & 4 & 46,00 & 3,00 & 1,00 \\
\hline 48 & 51 & 0 & 0 & 0 & 0 & 0 & 0,00 & 0,00 & $\overline{0,00}$ \\
\hline 51 & 54 & 1 & 51 & 8 & 1 & 9 & 51,00 & 8,00 & 1,00 \\
\hline 54 & 57 & 0 & 0 & 0 & 0 & 0 & 0,00 & 0,00 & 0,00 \\
\hline 57 & 60 & 0 & 0 & 0 & 0 & 0 & 0,00 & 0,00 & 0,00 \\
\hline 60 & 63 & 0 & 0 & 0 & 0 & 0 & 0,00 & 0,00 & 0,00 \\
\hline 63 & 66 & 0 & 0 & 0 & 0 & 0 & 0,00 & 0,00 & 0,00 \\
\hline 66 & 69 & 0 & 0 & 0 & 0 & 0 & 0,00 & 0,00 & 0,00 \\
\hline 69 & 72 & 0 & 0 & 0 & 0 & 0 & 0,00 & 0,00 & 0,00 \\
\hline 72 & 75 & 0 & 0 & 0 & 0 & 0 & 0,00 & 0,00 & $\overline{0,00}$ \\
\hline 75 & 78 & 1 & 75 & 6 & 1 & 7 & 75,00 & 6,00 & 1,00 \\
\hline 78 & 81 & 0 & 0 & 0 & 0 & 0 & 0,00 & 0,00 & 0,00 \\
\hline$\ldots$ & $\ldots$ & $\ldots$ & $\ldots$ & $\ldots$ & $\ldots$ & $\ldots$ & $\ldots$ & $\ldots$ & $\ldots$ \\
\hline 90 & 93 & 0 & 0 & 0 & 0 & 0 & 0,00 & 0,00 & 0,00 \\
\hline
\end{tabular}

have a high positive correlation for Long Method Average and Commit Average, and for God Class Average and Commit Average. Those correlations in the Eclipse project are lower than in the Tomcat project, even though both projects presented a significant correlation. When we consider both projects together, the results keep in-between, as expected.

\subsection{Answering the research questions}

As discussed in section 4.2, our main research question was split in two, each regarding the association between the occurrences of one code smell and the corresponding commits. Considering the observed high correlation, we cannot refute the hypothesis that the number of occurrences of Long Method may have a positive impact on the amount of commits. As for the God Class code smell, due to the observed moderate to low positive correlation, we have to say it the other way round: we cannot sustain the hypothesis that the number of occurrences of Long Method may have an impact on the amount of commits.

\subsection{Threats to Validity}

We are aware of the limitations of correlational analyses such as the used in this study. For this reason, we dubbed this paper as an exploratory study. The evidence raised basically allows to stand or discard hypotheses that must be tested, preferably with controlled experiments. The conclu- 
Table 3: Classes grouped by commit ranges (ECLIPSE JDT CORE)

\begin{tabular}{|c|c|c|c|c|c|c|c|c|c|}
\hline \multicolumn{1}{|l|}{ (I) } & (II) & (III) & (IV) & (V) & (VI) & (VII) & (VIII) & (IX) \\
\hline 0 & 3 & 279 & 227 & 199 & 109 & 308 & 0,81 & 0,71 & 0,39 \\
\hline 3 & 6 & 142 & 541 & 115 & 64 & 179 & 3,81 & 0,81 & 0,45 \\
\hline 6 & 9 & 80 & 548 & 81 & 42 & 123 & 6,85 & 1,01 & 0,53 \\
\hline 9 & 12 & 56 & 554 & 93 & 24 & 117 & 9,89 & 1,66 & 0,43 \\
\hline 12 & 15 & 41 & 521 & 91 & 22 & 113 & 12,71 & 2,22 & 0,54 \\
\hline 15 & 18 & 23 & 367 & 60 & 14 & 74 & 15,96 & 2,61 & 0,61 \\
\hline 18 & 21 & 17 & 325 & 43 & 11 & 54 & 19,12 & 2,53 & 0,65 \\
\hline 21 & 24 & 12 & 260 & 21 & 9 & 30 & 21,67 & 1,75 & 0,75 \\
\hline 24 & 27 & 14 & 344 & 84 & 7 & 91 & 24,57 & 6,00 & 0,50 \\
\hline 27 & 30 & 15 & 418 & 100 & 1 & 101 & 27,87 & 6,67 & 0,07 \\
\hline 30 & 33 & 4 & 124 & 14 & 4 & 18 & 31,00 & 3,50 & 1,00 \\
\hline 33 & 36 & 2 & 66 & 2 & 1 & 3 & 33,00 & 1,00 & 0,50 \\
\hline 36 & 39 & 1 & 37 & 1 & 2 & 3 & 37,00 & 1,00 & 2,00 \\
\hline 39 & 42 & 3 & 120 & 7 & 1 & 8 & 40,00 & 2,33 & 0,33 \\
\hline 42 & 45 & 0 & 0 & 0 & 1 & 1 & 0,00 & 0,00 & 0,00 \\
\hline 45 & 48 & 4 & 184 & 13 & 1 & 14 & 46,00 & 3,25 & 0,25 \\
\hline 48 & 51 & 0 & 0 & 0 & 0 & 0 & 0,00 & 0,00 & 0,00 \\
\hline 51 & 54 & 1 & 52 & 6 & 1 & 7 & 52,00 & 6,00 & 1,00 \\
\hline 54 & 57 & 0 & 0 & 0 & 0 & 0 & 0,00 & 0,00 & 0,00 \\
\hline 57 & 60 & 1 & 57 & 3 & 0 & 3 & 57,00 & 3,00 & 0,00 \\
\hline 60 & 63 & 0 & 0 & 0 & 0 & 0 & 0,00 & 0,00 & 0,00 \\
\hline 63 & 66 & 0 & 0 & 0 & 0 & 0 & 0,00 & 0,00 & 0,00 \\
\hline 66 & 69 & 0 & 0 & 0 & 0 & 0 & 0,00 & 0,00 & 0,00 \\
\hline 69 & 72 & 0 & 0 & 0 & 0 & 0 & 0,00 & 0,00 & 0,00 \\
\hline 72 & 75 & 0 & 0 & 0 & 0 & 0 & 0,00 & 0,00 & 0,00 \\
\hline 75 & 78 & 0 & 0 & 0 & 0 & 0 & 1 & 1 & 0,00 \\
\hline 78 & 81 & 1 & 79 & 11 & 0 & 11 & 79,00 & 11,00 & 0,00 \\
\hline 81 & 84 & 0 & 0 & 0 & 0 & 0 & 0,00 & 0,00 & 0,00 \\
\hline 84 & 87 & 0 & 0 & 0 & 0 & 0 & 0,00 & 0,00 & 0,00 \\
\hline 87 & 90 & 1 & 89 & 0 & 0 & 0 & 89 & 0,00 & 0,00 \\
\hline 90 & 93 & 0 & 0 & 0 & 0 & 0 & 0,00 & 0,00 & 0,00 \\
\hline & & & & & & & & & \\
\hline
\end{tabular}

sion validity is related to the ability to draw significant correct conclusions. For this reason, we applied the distribution adherence testing and the correlation analysis by commit ranges based on dispersion chats to support the analysis. We discussed the internal validity in terms of threats to the design of the study. For this reason, we managed to carefully plan the study and the variables to consider in the analysis as described in the six steps presented in Section 3 The use of the PMD tool for collecting code smells can be also a potential threat, since several authors have pointed out the inherent subjectivity in the code smells definition [23] can lead to unmatched detection, when using different tools. We expect to mitigate this last problem through our ongoing research work on the Crowdsmelling approach [24]. The external validity represents the possibility of generalizing the findings of this study. We identified external validity threats in our results, since the analysis was restricted to data from two open source software project systems, implemented in one programming language and focusing on two code smells from the catalogue published in [2]. However, the fact that these two open source software projects have an active community is an evidence of the representative-

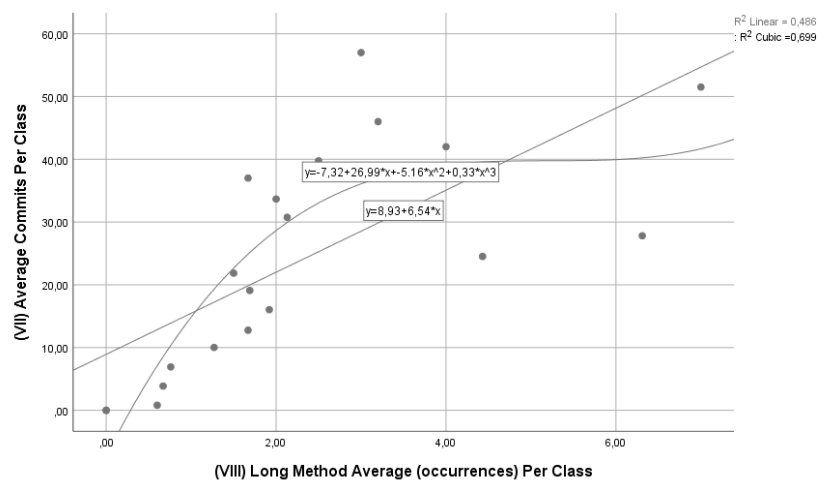

Figure 2: Dispersion Chart between Average Commits and Long Method Average occurrences (both projects)

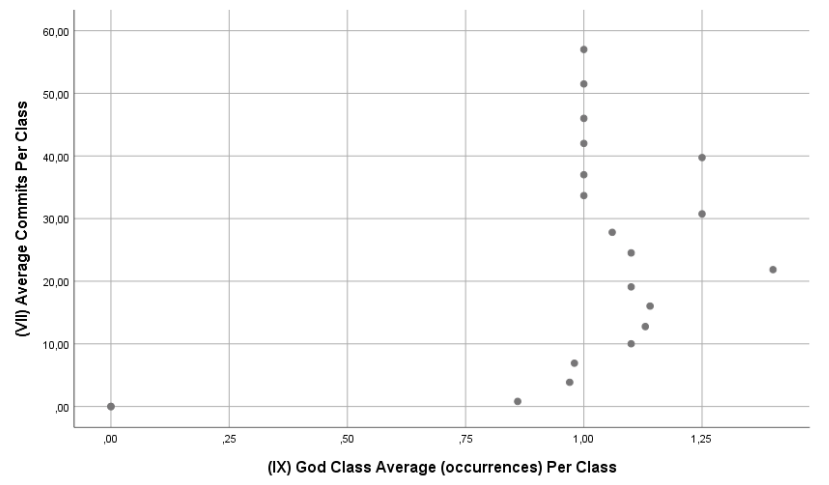

Figure 3: Dispersion Chart between Average Commits and God Class Average occurrences (both projects)

ness of these projects to support the conclusions drawn is this study for the analyzed code smells implemented in the Java language.

\section{Conclusions}

In this paper, we conducted an exploratory study upon two open source projects (Apache Tomcat and Eclipse JDT Core Component), in order to check if the occurrences of the Long Method and God Class code smells were associated to software defects (bugs). The PMD tool was applied to gather those code smells from all classes of those projects where code fixes occurred. The REST API of the Bugzilla tool was used to identify which were the files affected by code fixes (i.e. those where commits occurred during code fixing), by collecting data related to all closed bugs of those projects. We applied the Spearman correlation coefficient to evaluate the association between average values (per class) of a) occurrences of Long Method and observed commits; b) occurrences of God Classes and observed commits; and c) occurrences of both code smells and observed commits. We obtained a strong correlation of Long Method and Commits and revealead a poor correlation of God Class 
Table 4: Spearman correlations (code smells vs commit ranges)

\begin{tabular}{|c|c|c|}
\hline Project & $\begin{array}{c}\text { Long Method } \\
\text { average vs } \\
\text { Commit average }\end{array}$ & $\begin{array}{c}\text { God Class } \\
\text { average vs } \\
\text { Commit average }\end{array}$ \\
\hline Tomcat & $93 \%$ & $52 \%$ \\
\hline Eclipse & $72 \%$ & $30 \%$ \\
\hline Both & $85 \%$ & $33 \%$ \\
\hline
\end{tabular}

Table 5: Interpreting the Correlation Coefficient [22]

\begin{tabular}{|l|l|}
\hline Correlation & Interpretation of correlation \\
\hline $90 \%$ to $100 \%$ & Very high positive (negative) \\
\hline $70 \%$ to $90 \%$ & High positive (negative) \\
\hline $50 \%$ to $70 \%$ & Moderate positive (negative) \\
\hline $30 \%$ to $50 \%$ & Low positive (negative) \\
\hline $0 \%$ to $30 \%$ & Negligible correlation \\
\hline
\end{tabular}

and Commits, regarding both projects analyzed. Therefore, the higher is the number of Long Method, the higher is expected to be the number of commits in a class, although the effect is probably non-linear. In practice, we got strong evidence in those datasets that the occurrence of the Long Method may make the maintenance process harder, so we should avoid it through refactoring operations.

\section{References}

[1] L. Erlikh, "Leveraging legacy system dollars for e-business," IT professional, vol. 2, no. 3, pp. 17-23, 2000.

[2] M. Fowler, K. Beck, J. Brant, W. Opdyke, and D. Roberts, Refactoring: improving the design of existing code. AddisonWesley Professional, 1999.

[3] S. M. Olbrich, D. S. Cruzes, and D. I. K. SjÃäÿberg, "Are all code smells harmful? a study of god classes and brain classes in the evolution of three open source systems," in 2010 IEEE International Conference on Software Maintenance, Sep. 2010, pp. 1-10.

[4] A. Yamashita and L. Moonen, "To what extent can maintenance problems be predicted by code smell detection?-an empirical study," Information and Software Technology, vol. 55, no. 12, pp. 2223$2242,2013$.

[5] D. I. K. SjÃ ÿberg, A. Yamashita, B. C. D. Anda, A. Mockus, and T. Dyb ̃̌ě, "Quantifying the effect of code smells on maintenance effort," IEEE Transactions on Software Engineering, vol. 39, no. 8, pp. 1144-1156, Aug 2013.

[6] M. Lanza and R. Marinescu, Object-oriented metrics in practice: using software metrics to characterize, evaluate, and improve the design of object-oriented systems. $\quad$ Springer Science \& Business Media, 2007.

[7] H. Aman, "An empirical analysis on fault-proneness of wellcommented modules," in Empirical Software Engineering in Practice (IWESEP), 2012 Fourth International Workshop on. IEEE, 2012, pp. 3-9.

[8] F. Palomba, G. Bavota, M. Di Penta, F. Fasano, R. Oliveto, and A. De Lucia, "On the diffuseness and the impact on maintainability of code smells: a large scale empirical investigation," Empirical
Software Engineering, pp. 1-34, 2017

[9] A. Saboury, P. Musavi, F. Khomh, and G. Antoniol, "An empirical study of code smells in javascript projects," in Software Analysis, Evolution and Reengineering (SANER), 2017 IEEE 24th International Conference on. IEEE, 2017, pp. 294-305.

[10] M. M. Lehman, "Laws of software evolution revisited," in European Workshop on Software Process Technology. Springer, 1996, pp. 108-124.

[11] M. Tufano, F. Palomba, G. Bavota, R. Oliveto, M. Di Penta, A. De Lucia, and D. Poshyvanyk, "When and why your code starts to smell bad," in Proceedings of the 37th International Conference on Software Engineering-Volume 1. IEEE Press, 2015, pp. 403-414.

[12] G. Rasool and Z. Arshad, "A review of code smell mining techniques," Journal of Software: Evolution and Process, vol. 27, no. 11, pp. 867-895, 2015.

[13] E. Fernandes, J. Oliveira, G. Vale, T. Paiva, and E. Figueiredo, "A review-based comparative study of bad smell detection tools," in Proceedings of the 20th International Conference on Evaluation and Assessment in Software Engineering. ACM, 2016, p. 18.

[14] M. Mika, J. Vanhanen, C. Lassenius et al., "A taxonomy and an initial empirical study of bad smells in code," in null. IEEE, 2003, p. 381.

[15] W. Li and R. Shatnawi, "An empirical study of the bad smells and class error probability in the post-release object-oriented system evolution," Journal of systems and software, vol. 80, no. 7, pp. 11201128, 2007.

[16] F. Khomh, M. Di Penta, and Y.-G. Gueheneuc, "An exploratory study of the impact of code smells on software change-proneness," in Reverse Engineering, 2009. WCRE'09. 16th Working Conference on. IEEE, 2009, pp. 75-84.

[17] S. M. Olbrich, D. S. Cruzes, and D. I. Sjøberg, "Are all code smells harmful? a study of god classes and brain classes in the evolution of three open source systems," in Software Maintenance (ICSM), 2010 IEEE International Conference on. IEEE, 2010, pp. 1-10.

[18] R. Nascimento and C. Sant'Anna, "Investigating the relationship between bad smells and bugs in software systems," in Proceedings of the 11th Brazilian Symposium on Software Components, Architectures, and Reuse. ACM, 2017, p. 4.

[19] A. Cairo, G. Carneiro, and M. Monteiro, "The impact of code smells on software bugs: A systematic literature review," Information, vol. 9, no. 11, p. 273, 2018.

[20] V. Basili, G. Caldiera, and H. ROMBACH, "Goal question metric approach paradigm," pp. 528-532, 1994.

[21] N. Moha, Y.-G. Gueheneuc, A.-F. Duchien et al., "Decor: A method for the specification and detection of code and design smells," IEEE Transactions on Software Engineering (TSE), vol. 36, no. 1, pp. 2036,2010

[22] D. E. Hinkel, W. Wiersma, and S. G. Jurs, Applied statistics for the behavioral sciences, 5th ed. Hougthon Mifflin Company, 2003

[23] S. Bryton, F. Brito e Abreu, and M. Monteiro, "Reducing subjectivity in code smells detection: Experimenting with the long method," in 2010 Seventh International Conference on the Quality of Information and Communications Technology. IEEE, 2010, pp. 337-342.

[24] J. P. dos Reis, F. Brito e Abreu, and G. Carneiro, "Code smells detection 2.0: Crowdsmelling and visualization," in 2017 12th Iberian Conference on Information Systems and Technologies (CISTI). IEEE, 2017, pp. 1-4. 